\title{
Conditions for adiabatic spin transport in disordered systems
}

\author{
Markus Popp, ${ }^{1}$ Diego Frustaglia, ${ }^{2}$ and Klaus Richter ${ }^{1}$ \\ ${ }^{1}$ Institut für Theoretische Physik, Universität Regensburg, 93040 Regensburg, Germany \\ ${ }^{2}$ Institut für Theoretische Festkörperphysik, Universität Karlsruhe, 76128 Karlsruhe, Germany
}

(Received 7 March 2003; revised manuscript received 9 April 2003; published 11 July 2003)

\begin{abstract}
We address the controversy concerning the necessary conditions for the observation of Berry phases in disordered mesoscopic conductors. For this purpose, we calculate the spin-dependent conductance of disordered two-dimensional structures in the presence of inhomogeneous magnetic fields. Our numerical results show that for both, the overall conductance and quantum corrections, the relevant parameter defining adiabatic spin transport scales with the square root of the number of scattering events, in generalization of Stern's original proposal [A. Stern, Phys. Rev. Lett. 68, 1022 (1992)]. This could hinder a clear-cut experimental observation of Berry phase effects in diffusive metallic rings.
\end{abstract}

DOI: 10.1103/PhysRevB.68.041303

PACS number(s): 73.23.-b, 72.10.-d, 72.25.-b, 03.65.Vf

In contrast to phenomena related to Aharonov-Bohm (AB) phases ${ }^{2}$ for charge carriers, the corresponding observation of Berry phases ${ }^{3}$ due to the coupling of a spin to an orientationally nonuniform magnetic field $B$ requires the limit of adiabatic spin evolution. In mesoscopic conductors, such a limit corresponds to the situation where the carrier spin can follow the spatially varying field during transport through the system. In terms of time scales, the adiabatic limit is reached when the Larmor frequency of spin precession, $\omega_{\mathrm{s}}=2 \mu \mathrm{B} / \hbar$, is large compared to the reciprocal of a characteristic time scale $t_{\mathrm{c}}$ on which, from the point of view of the spin, the direction of the field has changed significantly during motion. There is a consensus that for ballistic (disorder-free) systems with magnetic-field configurations, commonly theoretically considered ${ }^{1,4-6}$ and experimentally realized, ${ }^{7} t_{\mathrm{c}} \sim L / v_{\mathrm{F}}$, where $v_{\mathrm{F}}$ denotes the Fermi velocity of the carriers and $L$ is the characteristic length scale of the system over which the field changes. For one-dimensional (1D) ballistic systems the condition for adiabaticity, $\omega_{\mathrm{s}}$ $\gg 2 \pi / t_{\mathrm{c}}$, therefore reads ${ }^{8}$

$$
Q_{1 \mathrm{D}} \equiv \frac{\omega_{\mathrm{s}}}{2 \pi v_{\mathrm{F}} / L} \gg 1,
$$

where we introduced the adiabaticity parameter $Q_{1 \mathrm{D}}$.

However, in the case of disordered systems, there are two candidates for the characteristic time $t_{\mathrm{c}}$ : (i) the mean elastic scattering time $\tau$ and (ii) the Thouless time $t_{\mathrm{Th}}=(L / \ell)^{2} \tau$, with $\ell=v_{\mathrm{F}} \tau$ as the elastic mean free path. The issue that which of these two time scales is the relevant one has recently led to a controversial discussion. ${ }^{9,10}$

In his proposal for 1D diffusive rings Stern ${ }^{1}$ perturbatively calculated the lifetime of the adiabatic eigenstates and compared it to $t_{\mathrm{Th}}$. He arrived at the condition

$$
Q_{1 \mathrm{D}} \gg L / \ell
$$

for adiabatic spin transport [Eq. (7) in Ref. 1]. This corresponds to setting $t_{c}=\tau$. Comparing Eqs. (1) and (2) one recognizes that in the diffusive regime, $L \gg \ell$, the adiabatic limit would require a magnetic field $L / \ell$ times larger than in the ballistic case. This "pessimistic criterion," which later has also been advocated by van Langen et al., ${ }^{9}$ would imply field strengths in the quantum Hall regime that let an experimental observation of Berry phases in diffusive metallic rings appear rather unlikely.

Alternatively, in analogy to the ballistic traveling time $L / v_{F}$, it appears convincing to associate $t_{\mathrm{c}}$ for diffusive systems with $t_{\mathrm{Th}}$, the time the electron takes to diffuse through the structure. This argumentation has been put forward by Loss and co-workers. ${ }^{4,10,11}$ By calculating the quantum corrections of the conductance in diffusive $1 \mathrm{D}$ rings, they predicted clear signatures of Berry's phase to be observable in a regime given by

$$
Q_{1 \mathrm{D}} \gg \ell / L .
$$

This condition for adiabaticity differs from criterion (2) by a factor $(L / \ell)^{2}$ and predicts for the observability of Berry phases a field strength above $20 \mathrm{mT},{ }^{11}$ which is well in the reach of modern experimental techniques. ${ }^{7}$

In view of various recent experimental efforts to observe Berry phases in the magneto conductance of mesoscopic rings, ${ }^{7,12-14}$ a clarification of the issue of the relevant time scale is desirable. The derivations of conditions (2) and (3) were based on diagrammatic and semiclassical techniques. Here, we choose a different approach and study numerically the spin-dependent conductance of ballistic and disordered mesoscopic systems in the presence of a spatially varying magnetic field $\vec{B}(\vec{r})=\vec{\nabla} \times \vec{A}(\vec{r})$. The Hamiltonian for noninteracting electrons with effective mass $m^{*}$ and charge $-e$ reads

$$
H=\frac{1}{2 m^{*}}\left[\vec{p}+\frac{e}{c} \vec{A}(\vec{r})\right]^{2}+V(\vec{r})+\mu \vec{B}(\vec{r}) \cdot \vec{\sigma} .
$$

The nontrivial coupling of the spin to the magnetic field enters via the Zeeman term $\mu \vec{B}(\vec{r}) \cdot \vec{\sigma}$, where $\vec{\sigma}$ is the Pauli spin vector and $\mu=g^{*} e \hbar /\left(4 m_{0} c\right)$ the magnetic moment with $g^{*}$ the gyromagnetic ratio. The electrostatic potential $V(\vec{r})$ includes the confinement and the potential of random impurities in the disordered case. At $T=0$, the spindependent conductance of a mesoscopic system with two attached leads is given by the Landauer formula ${ }^{15}$ 


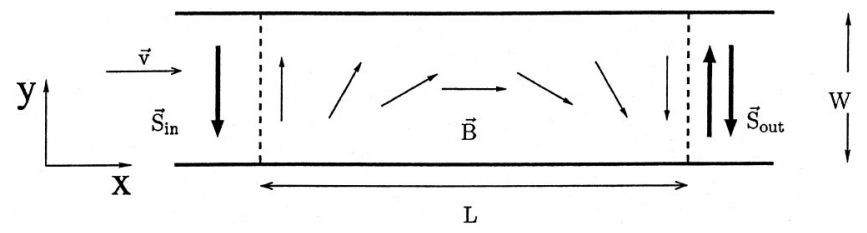

FIG. 1. 2D strip configuration used for the calculations of the spin-dependent conductance. The magnetic field $\vec{B}(x)$ performs a $180^{\circ}$ rotation within the plane of the strip. Spin states $\left(\vec{S}_{\text {in }}, \vec{S}_{\text {out }}\right)$ are defined with respect to the $y$ axis.

$$
G=\frac{e^{2}}{h} \sum_{s^{\prime}, s= \pm 1} T_{s^{\prime} s}=\frac{e^{2}}{h} \sum_{m^{\prime}, m=1}^{M} \sum_{s^{\prime}, s= \pm 1}\left|t_{s^{\prime} s}^{m^{\prime} m}\right|^{2} .
$$

Here, $t_{s^{\prime} s}^{m^{\prime} m}$ is the transmission amplitude from an incoming channel $m$ with spin $s$ to an outgoing channel $\left(m^{\prime}, s^{\prime}\right)$. We calculate $t_{s^{\prime} s}^{m^{\prime} m}$ by projecting the corresponding Greenfunction matrix onto the asymptotic spinors in the leads. We compute the Green function for Hamiltonian (4) numerically, using a generalized version of the recursive Green-function technique based on a tight-binding model ${ }^{15}$ including spin. ${ }^{16}$ We model the (nonmagnetic) disorder potential leading to elastic scattering within an Anderson model by choosing random $\delta$-like scatterers with amplitudes following a box distribution. The spin-dependent conductance is then obtained from ensemble averages over independent disorder configurations. $^{17}$

We now turn to the subject of interest and study how adiabaticity is approached in mesoscopic spin quantum transport. For this purpose, we introduce a model system consisting of a $2 \mathrm{D}$ strip with a rotating in-plane magnetic field between two ballistic leads, see Fig. 1. This system can also be regarded as a model for transport through magnetic domain walls. We assume incoming electrons with spin-down polarization in the $-y$ direction, ${ }^{18}$ injected from the left with Fermi wave number $k_{\mathrm{F}}=2 \pi / \lambda_{\mathrm{F}}$.

We first consider the overall conductance. In the ballistic case, it is feasible to derive an analytical expression for the spin-resolved transmission of this system using a transfermatrix approach. The normalized transmission $T_{\downarrow \downarrow}$ for spindown polarized incoming electrons to exit the system with spin-down polarization reads ${ }^{19}$

$$
T_{\downarrow \downarrow} \equiv \frac{1}{M} \sum_{m^{\prime}, m=1}^{M}\left|t_{-1,-1}^{m^{\prime} m}\right|^{2}=\sum_{m=1}^{M} \frac{\sin ^{2}\left(\frac{\pi}{2} \sqrt{1+Q_{m}^{2}}\right)}{M\left(1+Q_{m}^{2}\right)}
$$

with the generalized adiabaticity parameter [Eq. (1)]

$$
Q_{m} \equiv \frac{g^{*}}{k_{\mathrm{F}} W} \frac{m^{*}}{m_{0}}\left(\frac{L W B}{h c / e}\right)\left[1-\left(\frac{m \pi}{k_{\mathrm{F}} W}\right)^{2}\right]^{-1 / 2}
$$

for the $m$ th propagating mode in a 2D strip of length $L$ and width $W$. Summing over all transverse modes in Eq. (6), we find that the overall dependence of the ballistic transmission (dashed lines in Fig. 2) is given by a Lorentzian $T_{\downarrow \downarrow} \simeq 1 /(1$ $+Q^{2}$ ) (dotted lines). This defines an "effective" adiabaticity parameter $Q \sim B$ for the 2D strip, with $Q_{1}<Q<Q_{M}$ and $Q$

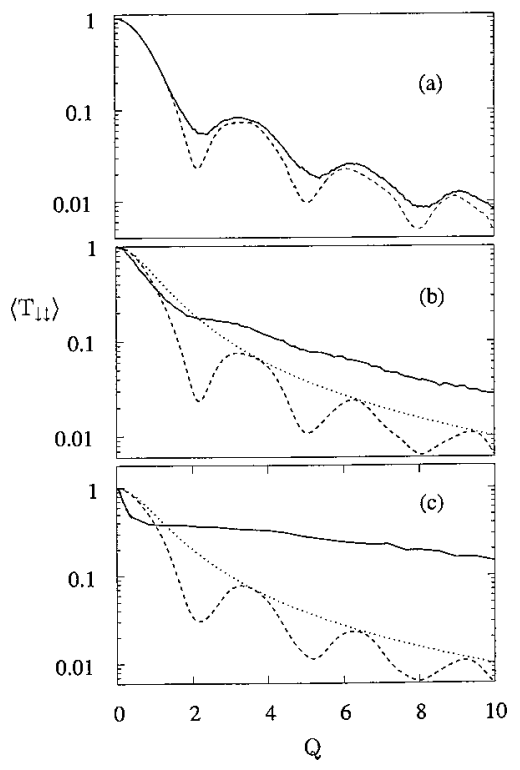

FIG. 2. Ensemble-averaged normalized transmission $\left\langle T_{\downarrow \downarrow}\right\rangle$ for a disordered (solid lines) and ballistic (dashed) 2D strip (Fig. 1) as a function of the adiabaticity parameter $Q \sim B$. The panels correspond to different disorder strengths: (a) quasiballistic; $L / \ell=0.5(L / W$ $=4.4)$; (b) moderate, $L / \ell=3(L / W=7.8)$; and (c) diffusive, $L / \ell$ $=10(L / W=13.9)$. The ballistic curves, Eq. (6), differ slightly from each other since they correspond to different Fermi wave vectors (ranging from $k_{\mathrm{F}} W / \pi=7.7$ to 11.6 ), but show the same overall Lorentzian decay with $Q$ (dotted).

$\sim 1.4 Q_{1 \mathrm{D}}$. This allows us to introduce a quantity that solely characterizes the adiabatic regime in the case of several open channels.

For $B \rightarrow 0$, the spin direction is preserved and $T_{\downarrow \downarrow}$ is maximal. In the limit of a strong $B$ field, the spin stays adiabatically aligned with the orientationally inhomogeneous field during transport, minimizing the probability of leaving the conductor in Fig. 1 in a spin-down state. The Lorentzian dependence of $T_{\downarrow \downarrow}$ on $Q \sim B$ reflects this behavior and appears as the natural measure for the crossover from the nonadiabatic $\left(T_{\downarrow \downarrow} \rightarrow 1, Q \ll 1\right)$ to the adiabatic $\left(T_{\downarrow \downarrow} \rightarrow 0, Q \gg 1\right)$ regime.

To find a proper condition for adiabaticity in the disordered case we compute the ensemble averaged transmission $\left\langle T_{\downarrow \downarrow}\right\rangle$ in the presence of elastic scattering for $\lambda_{\mathrm{F}} \ll \ell$ as a function of $Q$ and compare it to the ballistic result (6). Our results for different ratios $L / \ell$ are shown as the solid lines in Fig. 2 exhibiting the following features.

(i) The oscillations in the ballistic transmission are averaged out with increasing disorder.

(ii) For $Q \gg 1$, the normalized $\left\langle T_{\downarrow \downarrow}\right\rangle$ is larger in the disordered than in the ballistic case. This means that in the presence of elastic scattering a stronger scaled field $Q$ is required for acceding to the adiabatic regime of $\left\langle T_{\downarrow \downarrow}\right\rangle \approx 0$.

(iii) For $Q \ll 1$ we observe the opposite behavior: The non-adiabatic limit of almost maximum transmission $\left\langle T_{\downarrow \downarrow}\right\rangle$ is restricted to lower magnetic fields compared to the ballistic case.

(iv) The crossover region $Q \sim 1$ is characterized by a 


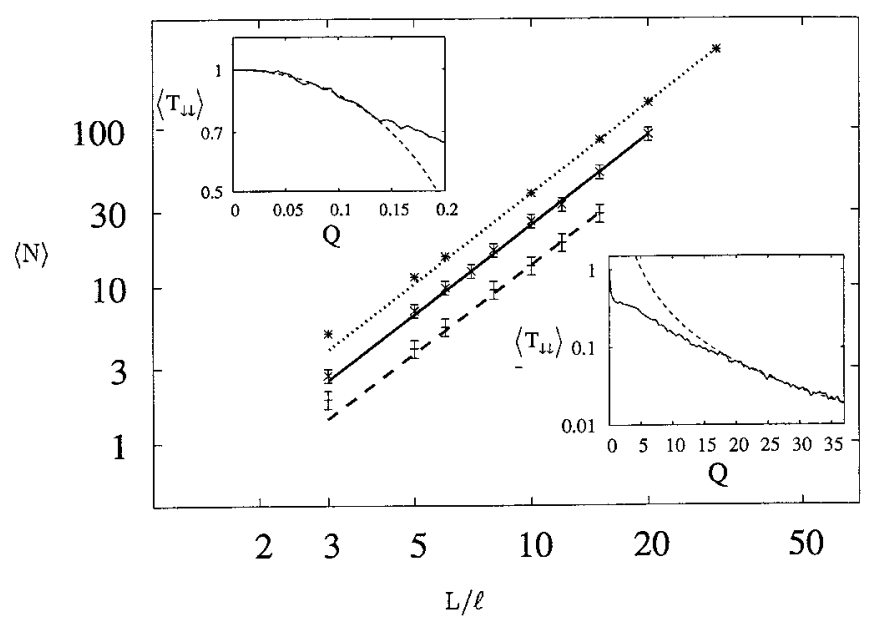

FIG. 3. Functional dependence of fit parameters $N_{\text {ad }}$ (solid line) and $N_{\text {nad }}$ (dashed) on the scaled length $L / \ell$ of the disordered strip. Linear regression yields $N_{\text {ad }}=0.31(L / \ell)^{1.9}$ (solid line) and $N_{\text {nad }}$ $=0.18(L / \ell)^{1.88}$ (dashed). For comparison, the mean number of scattering events $\langle N\rangle=0.48(L / \ell)^{1.91}$ is also shown (dotted), obtained from an independent $1 \mathrm{D}$ random-walk model. Insets: Transmission $\left\langle T_{\downarrow \downarrow}\right\rangle$ and fitted Lorentzians in the nonadiabatic (left) and adiabatic (right) limit for strip with $L / \ell=10$. (Error bars include uncertainty from fitting procedure.)

transmission plateau, which approaches $\left\langle T_{\downarrow \downarrow}\right\rangle \approx\left\langle T_{\uparrow \downarrow}\right\rangle \approx 0.5$ with increasing diffusiveness. Here, the nonmagnetic disorder acts as a spin randomizer of the originally spin-polarized current. The features (i) -(iv) already begin to appear in the quasiballistic regime [Fig. 2(a)] and become more pronounced with increasing degree of diffusiveness given by the ratio $L / \ell$ [Fig. 2(c)].

After this qualitative discussion we now derive a quantitative condition for adiabaticity in the disordered strip. In analogy to the ballistic case, we expect the disorder averaged transmission $\left\langle T_{\downarrow \downarrow}\right\rangle$ to exhibit a scaled Lorentzian dependence in the limits of small and large $Q$. Indeed, in the adiabatic limit the Lorentz function is an excellent fit to the diffusive curve $\left\langle T_{\downarrow \downarrow}\right\rangle$ in Fig. 2(c), if the ballistic parameter $Q$ is replaced by $Q / \sqrt{N_{\text {ad }}}$ with $N_{\text {ad }}$ being fitted (left inset Fig. 3). Corresponding results hold for the nonadiabatic limit where we use the scaling $Q \sqrt{N_{\text {nad }}}$ (right inset in Fig. 3). We further determined $N_{\text {ad }}$ and $N_{\text {nad }}$ for various ratios $L / \ell$ and obtain power-law dependences illustrated in Fig. 3. We can, hence, formulate as a necessary condition for adiabatic spin transport through the disordered 2D strip: $Q \gg(L / \ell)^{0.95}$. Comparing this with Eq. (2) we obtain a smaller exponent. To explain this deviation, we note that Eq. (2) can be written in the more general form $Q \gg \sqrt{\langle N\rangle}$, with the average number of scattering events $\langle N\rangle=\left\langle t_{\mathrm{Th}}\right\rangle / \tau=(L / \ell)^{2}$. This suggests to associate $N_{\text {ad }}$ and $N_{\text {nad }}$ with the number of scattering events the electron has to undergo upon traversing the microstructure. Due to the strong coupling of the finite-size 2D strip to the ballistic leads, we expect the diffusion time to be smaller than the Thouless time $t_{\mathrm{Th}}$, thus reducing the number of scattering events.

To confirm the above arguments we, independently, checked numerically the dependence of $\langle N\rangle$ on the scaled length $L / \ell$ of a finite disorder conducting strip. To this end,

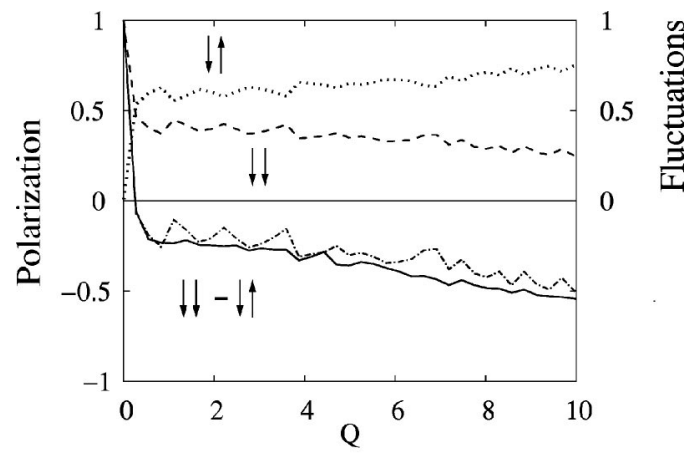

FIG. 4. Quantum fluctuations $\delta g_{\downarrow \downarrow} /\left(\delta g_{\downarrow \downarrow}+\delta g_{\uparrow \downarrow}\right)$ (dashed) and $\delta g_{\uparrow \downarrow} /\left(\delta g_{\downarrow \downarrow}+\delta g_{\uparrow \downarrow}\right)$ (dotted) as a function of $Q$ for a diffusive strip with $L / \ell=15$. For comparison, we also show ( $\delta g_{\downarrow \downarrow}$ $\left.-\delta g_{\uparrow \downarrow}\right) /\left(\delta g_{\downarrow \downarrow}+\delta g_{\uparrow \downarrow}\right) \quad$ (dashed-dotted) and the polarization $\left[\left\langle T_{\downarrow \downarrow}\right\rangle-\left\langle T_{\uparrow \downarrow}\right\rangle\right] /\left[\left\langle T_{\downarrow \downarrow}\right\rangle+\left\langle T_{\uparrow \downarrow}\right\rangle\right]$ (solid).

we used a 1D (Ref. 20) random-walk model taking into account explicitly the interface between the disordered and ballistic regions. We find that $\langle N\rangle$ obeys a power law in $L / \ell$ with exponent 1.91 which, as expected, is lower than two (dotted line in Fig. 3). In Fig. 3, besides small deviations for small $L / \ell$ in the nondiffusive limit, there is a good agreement with the fitted straight lines for $L / \ell \geqslant 5$, indicating a diffusive behavior. Within the given error tolerance, all three curves in Fig. 3 exhibit identical exponents and deviate only in the prefactor of order one.

We conclude from our numerical, quantum-mechanical results, together with the expression for $\langle N\rangle$ from the independent random-walk model, that the adiabaticity parameter scales with $\sqrt{\langle N\rangle}$. This enables us to formulate a general (system-independent) adiabaticity condition for diffusive systems, which only depends on the corresponding adiabaticity parameter $Q$ of the ballistic system and the mean number of scattering events $\langle N\rangle:^{21}$

$$
Q \gg \sqrt{\langle N\rangle}
$$

For diffusive 1D rings, this criterion is in perfect agreement with Stern's original condition (2).

So far, we considered the total conductance dominated by the Boltzmann contribution. However, signatures of Berry phases in diffusive conductors appear only in the phase coherent part of the conductance, i.e., quantum corrections such as $\mathrm{AB}$ oscillations and universal conductance fluctuations (UCF's). To decide whether distinct Berry phase effects, e.g., in diffusive rings, can be observed at realistic magnetic-field strengths, one has to check if an adiabaticity condition, different from Eq. (8), holds for the quantum corrections. For this purpose, spin-resolved UCF's represent a suitable quantity, defined as $\delta g_{s^{\prime} s}=\sqrt{\left\langle T_{s^{\prime} s}^{2}\right\rangle-\left\langle T_{s^{\prime} s}\right\rangle^{2}}$ in units of $e^{2} / h$. We calculated $\delta g_{s^{\prime} s}$ numerically as a function of $Q$ for a diffusive 2D strip with $L / \ell=15$. The results are depicted in Fig. 4 in terms of the normalized difference $\left(\delta g_{\downarrow \downarrow}-\delta g_{\uparrow \downarrow}\right) /\left(\delta g_{\downarrow \downarrow}+\delta g_{\uparrow \downarrow}\right)$ which can be regarded as a polarization. We note that the UCF's exhibit precisely the same scaling behavior as the corresponding quantity for the total 
conductance, $\left(T_{\downarrow \downarrow}-T_{\uparrow \downarrow}\right) /\left(T_{\downarrow \downarrow}+T_{\uparrow \downarrow}\right)$, and consequently obey condition (8). Figure. 4 also illustrates the important fact that in a wide region $\ell / L \leqq Q \leqq L / \ell$, the respective adiabatic $\left(T_{\uparrow \downarrow}, \delta g_{\uparrow \downarrow}\right)$ and nonadiabatic $\left(T_{\downarrow \downarrow}, \delta g_{\downarrow \downarrow}\right)$ components are comparable in magnitude.

We further note that numerical quantum calculations of the spin-dependent magneto-conductance in disordered rings subject to a circular inhomogeneous $B$ field show that signatures of Berry phases appear only in the $\mathrm{AB}$ oscillations of the adiabatic components $\left(\left\langle T_{\uparrow \downarrow}\right\rangle\right.$ and $\left.\delta g_{\uparrow \downarrow}\right)$, which are dominated by electrons with spin always aligned with the local field. ${ }^{22}$ In view of Fig. 4, we can hence conclude for diffusive rings that in the experimentally relevant plateau region $\ell / L \leqq Q<1$, the adiabatic components, which show Berry phase signatures, are of the same magnitude as the nonadiabatic components. Berry phase effects in the $\mathrm{AB}$ oscillations of the total conductance and the UCF's are hence masked by the regular, nonadiabatic contribution. ${ }^{23}$ However, in this broad plateau region one still finds effects of the inhomogeneous magnetic field that can be ascribed to the nonadiabatic generalization of the Berry phase, the Aharonov-Anandan phase. ${ }^{24}$ Our numerical results imply that to observe clear Berry phase effects such as the "magic angles" found by Engel and Loss ${ }^{11}$ in the magnetoconductance and UCF's of diffusive rings, one has to go to the truly adiabatic regime given by condition (8).

For a typical experimental $\mathrm{AB}$ setup based on copper rings with radius $r_{0}=500 \mathrm{~nm}$ and $\ell=15 \mathrm{~nm}$ (Ref. 12), strict application of criterion (8) corresponds to $B$-field strengths larger than $10^{3} \mathrm{~T}$. On the other hand, according to the condition $Q \ll 1 / \sqrt{\langle N\rangle}$, the opposite nonadiabatic regime $\left(\left\langle T_{\downarrow \downarrow}\right\rangle\right.$ $\sim 1$ ) is restricted to fields smaller than $0.1 \mathrm{~T}$. In the broad intermediate $B$-field range, covering four orders of magnitude, the magnetoconductance is expected to show at most the signatures of the Aharonov-Anandan phase.

Recently, imprints of Berry's phase in $\mathrm{AB}$ oscillations have been reported for holes in quasiballistic 2D GaAs rings with strong spin-orbit interaction. ${ }^{14}$ A rough estimate of the system parameters suggests that the experimental conditions may fulfill the adiabaticity criterion (8) with $B$ replaced by an effective Rashba field strength.

To summarize, we studied spin-dependent quantum transport through 2D disordered geometries. We showed that the relevant parameter defining the adiabatic limit both for the total conductance and the quantum corrections scales with the square root of the number of scattering events. This can be cast into a generalized criterion for adiabaticity for both ballistic and disordered systems. It appears as a severe obstacle for direct experimental observation of Berry phases in the conductance through diffusive metal rings. Our numerical findings indicate that elastic scattering due to nonmagnetic impurities in the presence of a spatially varying magnetic field exhibits features similar to those in systems with spin flips associated with the scattering process as for magnetic impurities or Rashba spin-orbit coupling.

\section{ACKNOWLEDGMENTS}

We would like to thank H.-A. Engel and D. Loss for helpful discussions and acknowledge support from the Deutsche Forschungsgemeinschaft.
${ }^{1}$ A. Stern, Phys. Rev. Lett. 68, 1022 (1992).

${ }^{2}$ Y. Aharonov and D. Bohm, Phys. Rev. 115, 485 (1959).

${ }^{3}$ M.V. Berry, Proc. R. Soc. London, Ser. A 392, 45 (1984).

${ }^{4}$ D. Loss, H. Schöller, and P.M. Goldbart, Phys. Rev. B 48, 15218 (1993).

${ }^{5}$ D. Frustaglia and K. Richter, Found. Phys. 31, 399 (2001).

${ }^{6}$ D. Frustaglia, M. Hentschel, and K. Richter, Phys. Rev. Lett. 87, 256602 (2001).

${ }^{7}$ Transport through ballistic rings surrounding micromagnets has been reported by P. D. Ye, S. Tarucha, and D. Weiss, in Proceedings of the 24th ICPS, Jerusalem, edited by D. Gershoni (World Scientific, Singapore, 1999); transport through diffusive rings surrounding micromagnets has been reported by T.M. Jacobs and N. Giordano, Superlattices Microstruct. 23, 635 (1998).

${ }^{8}$ See Ref. 5 for adiabaticity in 2D ballistic structures.

${ }^{9}$ S.A. van Langen, H.P.A. Knops, J.C.J. Paasschens, and C.W.J. Beenakker, Phys. Rev. B 59, 2102 (1999).

${ }^{10}$ D. Loss, H. Schöller, and P.M. Goldbart, Phys. Rev. B 59, 13328 (1999).

${ }^{11}$ H.-A. Engel and D. Loss, Phys. Rev. B 62, 10238 (2000).

${ }^{12}$ R. Häußler, Ph.D. thesis, Universität Karlsruhe, 1999.

${ }^{13}$ A.F. Morpurgo, J.P. Heida, T.M. Klapwijk, B.J. van Wees, and G. Borghs, Phys. Rev. Lett. 80, 1050 (1998).

${ }^{14}$ J.-B. Yau, E.P. De Poortere, and M. Shayegan, Phys. Rev. Lett. 88, 146801 (2002).

${ }^{15}$ See, e.g., S. Ferry and S. M. Goodnick, Transport in Nanostruc- tures (Cambridge University Press, Cambridge, 1997).

${ }^{16}$ This has proven to be an efficient method for studying spindependent quantum transport, see Ref. 6 and D. Frustaglia, Ph.D. thesis, TU, Dresden, 2001.

${ }^{17}$ Testing this model on a narrow wire we find quantitative agreement with predictions for quantum conductance corrections (weak localization, universal conductance fluctuations) and an exponential decrease of the conductance when approaching the localized regime.

${ }^{18}$ Equivalent results are obtained for spin-up electrons.

${ }^{19}$ See Ref. 6 for a related derivation and M. Popp, Diploma thesis, Universität Regensburg, 2002.

${ }^{20}$ The restriction to a $1 \mathrm{D}$ model is justified since we are dealing with narrow strips of width $W \lesssim \ell$.

${ }^{21}$ To gain further insight into the scaling law (8), we developed a phenomenological model based on a perturbative approach valid in the far adiabatic and nonadiabatic limit. Assuming that spin flips before and after a scattering event are independent processes, we can derive a scaling behavior which coincides with condition (8) (Ref. 22). The ballistic limit then corresponds to $N=1$.

${ }^{22}$ M. Popp, D. Frustaglia, and K. Richter (unpublished).

${ }^{23}$ This has been numerically confirmed for the magnetoconductance in disordered $(L / \ell \approx 4)$ rings subject to an inhomogeneous field (Ref. 22).

${ }^{24}$ Y. Aharonov and J. Anandan, Phys. Rev. Lett. 58, 1593 (1987). 